\title{
THICK RESTARTING THE WEIGHTED HARMONIC GOLUB-KAHAN-LANCZOS ALGORITHM FOR THE LINEAR RESPONSE EIGENVALUE PROBLEM*
}

\author{
HONG-XIU ZHONG ${ }^{\dagger}$ AND GUO-LIANG CHEN ${ }^{\ddagger}$
}

\begin{abstract}
In this paper, we propose a weighted harmonic Golub-Kahan-Lanczos algorithm for the linear response eigenvalue problem (LREP). Convergence properties are established for the error bounds of the approximate eigenpairs. Moreover, we consider a practical thick-restart procedure to reduce the computational and memory costs and present a weighted harmonic Golub-Kahan-Lanczos algorithm with deflated restarting. Numerical tests show the efficiency of our new algorithms.
\end{abstract}

Key words. linear response eigenvalue problem, harmonic Rayleigh-Ritz projection, weighted Golub-KahanLanczos algorithm, thick restart

AMS subject classifications. 65F15, 65F50, 15A18

1. Introduction. In this paper, we consider the following eigenproblem

$$
\mathbf{H} z:=\left[\begin{array}{cc}
0 & K \\
M & 0
\end{array}\right]\left[\begin{array}{l}
u \\
v
\end{array}\right]=\lambda\left[\begin{array}{l}
u \\
v
\end{array}\right]=\lambda z
$$

where $K, M \in \mathbb{R}^{n \times n}$ are both symmetric and positive definite. Such a problem is referred to as linear response eigenvalue problem (LREP) [3, 4, 17, 24]. It describes the excitation states (energies) of physical systems in the study of the collective motion of many-particle systems. It is also known as the Bethe-Salpeter eigenvalue problem [19] or the random phase approximation eigenvalue problem [15]. The LREP has important applications in the area of computational quantum chemistry and physics such as silicon nanoparticles and nanoscale materials and the analysis of interstellar clouds and polarizabilities [3, 17].

All the eigenvalues of $\mathbf{H}$ are real and appear in pairs $\{\lambda,-\lambda\}$ [3,27], and we denote the ordered eigenvalues of $\mathbf{H}$ by

$$
-\lambda_{1} \leq \cdots \leq-\lambda_{n}<\lambda_{n} \leq \cdots \leq \lambda_{1} \quad \text { with } \quad \lambda_{j}>0(j=1, \ldots, n) .
$$

If either $K$ or $M$ or both are semidefinite, then some eigenvalues may be equal to zero [3]. Although there are cases that either $K$ or $M$ or both may be semidefinite [14], in most manyparticle systems, both $K$ and $M$ are positive definite [16, 17,23]. Thus throughout this paper, we consider the common case that both $K$ and $M$ are real symmetric positive definite. All the results in this paper can be simply extended to the complex Hermitian case.

Recently, an important notion for the LREP was proposed in [3], namely, the pair of deflating subspaces $\{\mathcal{U}, \mathcal{V}\} \subseteq \mathbb{R}^{n}$ which satisfy

$$
K \mathcal{U} \subseteq \mathcal{V} \quad \text { and } \quad M \mathcal{V} \subseteq \mathcal{U}
$$

The notion is fundamental for several efficient algorithms, e.g., the locally optimal block preconditioned four-dimensional conjugate gradient method [4], the block Chebyshev-Davidson method [22], and the generalized Lanczos method [21, 24]. All of the above methods generate

\footnotetext{
* Received October 30, 2018. Accepted November 11, 2019. Published online on December 16, 2019. Recommended by Michiel Hochstenbach.

${ }^{\dagger}$ School of Science, Jiangnan University, Wuxi, Jiangsu, 214122, P. R. China (zhonghongxiu @126. com).

${ }^{\ddagger}$ School of Mathematical Sciences, Shanghai Key Laboratory of PMMP, East China Normal University, Shanghai, 200241, P. R. China (gl chen@math. ecnu . edu . cn).
} 
approximate deflating subspaces and then approximate eigenpairs of $\mathbf{H}$ from a far smaller matrix $\mathbf{H}_{S}=\left[\begin{array}{cc}0 & K_{S} \\ M_{S} & 0\end{array}\right]$, where $K_{S}$ and $M_{S}$ are both symmetric and positive definite. Some other effective methods and a rigorous theoretical analysis can be found in the references [9, 26].

Motivated by the bidiagonalization idea in [1], a weighted Golub-Kahan-Lanczos method (wGKL) was proposed for solving the LREP in [28]. It aims to generate a projection matrix $\mathbf{B}_{k}=\left[\begin{array}{cc}0 & B_{k}^{T} \\ B_{k} & 0\end{array}\right]$ of $\mathbf{H}$ at the $k$ th iteration, where $B_{k}$ is an upper or lower bidiagonal matrix. Due to the symmetry of $\mathbf{B}_{k}$, the eigenpairs of $\mathbf{H}$ can be constructed just from $B_{k}$, not the whole $\mathbf{B}_{k}$. The convergence analysis shows that running $k$ iterations of wGKL is equivalent to applying $2 k$ iterations of a weighted Lanczos algorithm to $\mathbf{H}$ [28]. In the following discussion, we focus on the case that $B_{k}$ is an upper bidiagonal matrix; the lower bidiagonal case can be treated similarly.

It is well known that the classical Rayleigh-Ritz projection methods are suited to solve the exterior eigenproblems [18, 20], while harmonic Rayleigh-Ritz projection methods are often used as efficient techniques for interior eigenproblems [10, 11, 12, 25, 29]. If we want to find the eigenpairs of a matrix $A$, then the harmonic Arnoldi method seeks $(\theta, x)$ satisfying

$$
\left\{\begin{array}{l}
x \in \mathcal{K}_{m}(A, v), \\
A x-\theta x \perp(A-\sigma I) \mathcal{K}_{m}(A, v),
\end{array}\right.
$$

where $\sigma$ is the target, $\mathcal{K}_{m}(A, v)$ is the Krylov subspace, and $v$ is the initial vector. This pair $(\theta, x)$ is a so-called harmonic Ritz pair. In this paper, the interior eigenvalues of $\mathbf{H}$ that we seek are those near to 0, i.e., here $\sigma=0$. However, for the LREP, it is not known whether or not the harmonic type of WGKL is better suited for computing the interior eigenvalues. With WGKL, we propose a weighted harmonic Golub-Kahan-Lanczos algorithm (WHGKL) to solve the eigenproblem for $\mathbf{H}$ and study the question which one of WGKL and WHGKL methods is better for computing exterior or interior eigenvalues for LREP.

In this method, by increasing the iteration number, the dimension of the search space generally increases, thus, the computational cost of the orthogonalization usually becomes too large. This makes restarting necessary for solving interior eigenvalues. Motivated by the idea of the restarting strategy in $[2,13]$, in this paper, we consider how to restart our weighted harmonic Golub-Kahan-Lanczos algorithm using the deflated restarting strategy, i.e., we add some harmonic Ritz vectors to the search subspace to achieve the purpose of acceleration. Consequently, we propose a weighted harmonic Golub-Kahan-Lanczos with thick restarting algorithm (WHGKL-TR) for the LREP.

The rest of this paper is organized as follows. In Section 2, some preliminaries are provided. In Section 3, we propose our weighted harmonic Golub-Kahan-Lanczos algorithm and a second version, both for the LREP, and give their error bounds for the approximate eigenpairs. We also analyze which method is better for interior eigenvalues. In Section 4, we consider how to restart the weighted harmonic Golub-Kahan-Lanczos algorithm and present the main algorithm of this paper. Numerical experiments in Section 5 illustrate the benefits of our new algorithms.

Throughout this paper, we use a capital letter to represent a matrix, and we write $A_{i, j}$ for a matrix with $i$ rows and $j$ columns and write $A_{i}$ for a matrix with $i$ columns. $I_{n}$ is the $n \times n$ identity matrix. The vector $e_{j}$ is the $j$ th column of the identity matrix of suitable size. If there is no special explanation, an eigenvector is a right eigenvector, and the same rule applies to a (harmonic) Ritz vector. $A>0(\geq 0)$ means that the matrix $A$ is symmetric positive definite (semidefinite). We use $\|\cdot\|_{2}$ to express the spectral norm for matrices and the 2-norm for vectors. We use the weighted inner product $(x, y)_{A}=x^{T} A y$ in $\mathbb{R}^{n}$ obtained from an $n \times n$ symmetric positive definite matrix $A$. The corresponding weighted norm, called 
$A$-norm, is defined by $\|x\|_{A}=\sqrt{(x, x)_{A}}$. A matrix $X$ is $A$-orthonormal if $X^{T} A X=I$ (and it is $A$-orthogonal if $X$ is a square matrix). A set of vectors $\left\{x_{1}, \ldots, x_{k}\right\}$ is also called $A$-orthonormal if $X=\left[x_{1}, \ldots, x_{k}\right]$ is $A$-orthonormal and $A$-orthogonal if $\left(x_{i}, x_{j}\right)_{A}=0$ for $i \neq j$.

2. Preliminaries. In this section, we first introduce the weighted Golub-Kahan-Lanczos algorithm (wGKL) [28], which is a useful method for the LREP. For the symmetric and positive definite matrices $K$ and $M$ given in (1.1), there exists an $M$-orthogonal matrix $X \in \mathbb{R}^{n \times n}$ and a $K$-orthogonal matrix $Y \in \mathbb{R}^{n \times n}$ such that

$$
M X=Y B, \quad K Y=X B^{T},
$$

where $B$ is upper bidiagonal; for details see [28, Lemma 1]. Based on the relations in (2.1), the WGKL algorithm can be stated as follows.

ALGORITHM 1 (WGKL).

Choose $x_{1}$ satisfying $\left\|x_{1}\right\|_{M}=1$, and set $\beta_{0}=1, y_{0}=0$. Compute $g_{1}=M x_{1}$.

For $j=1,2, \ldots$

$$
\begin{aligned}
& s_{j}=g_{j} / \beta_{j-1}-\beta_{j-1} y_{j-1} \\
& f_{j}=K s_{j} \\
& \alpha_{j}=\left(s_{j}^{T} f_{j}\right)^{\frac{1}{2}} \\
& y_{j}=s_{j} / \alpha_{j} \\
& t_{j+1}=f_{j} / \alpha_{j}-\alpha_{j} x_{j} \\
& g_{j+1}=M t_{j+1} \\
& \beta_{j}=\left(t_{j+1}^{T} g_{j+1}\right)^{\frac{1}{2}} \\
& x_{j+1}=t_{j+1} / \beta_{j}
\end{aligned}
$$

End

Let

$$
X_{k}=\left[x_{1}, \ldots, x_{k}\right], \quad Y_{k}=\left[y_{1}, \ldots, y_{k}\right], \quad B_{k}=\left[\begin{array}{cccc}
\alpha_{1} & \beta_{1} & & \\
& \ddots & \ddots & \\
& & \ddots & \beta_{k-1} \\
& & \alpha_{k}
\end{array}\right],
$$

then after $k$ iterations, the following wGKL relations are valid:

$$
\begin{aligned}
M X_{k} & =Y_{k} B_{k}, \\
K Y_{k} & =X_{k} B_{k}^{T}+\beta_{k} x_{k+1} e_{k}^{T}=X_{k+1}\left[\begin{array}{ll}
B_{k} & \beta_{k} e_{k}
\end{array}\right]^{T} \triangleq X_{k+1} B_{k, k+1}^{T}, \\
X_{k}^{T} M X_{k} & =I_{k}=Y_{k}^{T} K Y_{k} .
\end{aligned}
$$

The wGKL process can be used to solve the LREP. One first defines

$$
\mathbf{X}_{k}=\left[\begin{array}{cc}
X_{k} & 0 \\
0 & Y_{k}
\end{array}\right] \quad \text { and } \quad \mathbf{B}_{k}=\left[\begin{array}{cc}
0 & B_{k}^{T} \\
B_{k} & 0
\end{array}\right]
$$

Then from (2.2),

$$
\mathbf{H X}_{k}=\mathbf{X}_{k} \mathbf{B}_{k}+\beta_{k}\left[\begin{array}{c}
x_{k+1} \\
0
\end{array}\right] e_{2 k}^{T} .
$$

Consequently, the first $\ell$ smallest eigenvalues of $\mathbf{H}$ together with their corresponding eigenvectors can be approximately constructed from $\mathbf{B}_{k}$, which is obviously symmetric. Suppose 
that $B_{k}$ has an SVD, $B_{k}=\bar{\Phi}_{k} \bar{\Sigma}_{k} \bar{\Psi}_{k}^{T}$, where $\bar{\Phi}_{k}=\left[\bar{\phi}_{1}, \ldots, \bar{\phi}_{k}\right], \bar{\Psi}_{k}=\left[\bar{\psi}_{1}, \ldots, \bar{\psi}_{k}\right]$, and $\bar{\Sigma}_{k}=\operatorname{diag}\left(\bar{\sigma}_{1}, \ldots, \bar{\sigma}_{k}\right)$, with $\bar{\sigma}_{1} \geq \cdots \geq \bar{\sigma}_{k}>0$. Then from (2.4), we may take

$$
\pm \bar{\lambda}_{j}= \pm \bar{\sigma}_{j}, \quad \bar{z}_{j}^{ \pm}=\frac{1}{\sqrt{2}}\left[\begin{array}{c}
X_{k} \bar{\psi}_{j} \\
\pm Y_{k} \bar{\phi}_{j}
\end{array}\right], \quad j=1, \ldots, k,
$$

as Ritz values along with the corresponding $\mathbf{M}$-orthonormal Ritz vectors of $\mathbf{H}$, where $\mathbf{M}=\left[\begin{array}{cc}M & 0 \\ 0 & K\end{array}\right]$. We may use the residual norm

$$
\left\|\mathbf{H} \bar{z}_{j}^{+}-\bar{\lambda}_{j} \bar{z}_{j}^{+}\right\|_{\mathbf{M}}=\frac{\beta_{k}\left|\bar{\phi}_{j k}\right|}{\sqrt{2}}
$$

as the stopping criteria, where $\bar{\phi}_{j k}$ is the $k$ th element of $\bar{\phi}_{j}$. The following algorithm is the weighted Golub-Kahan-Lanczos algorithm for the LREP [28], denoted by WGKL-1.

ALGORITHM 2 (WGKL-1).

1. Given an initial guess $x_{1}$ satisfying $\left\|x_{1}\right\|_{M}=1$, a tolerance tol, as well as $\ell$ the number of desired eigenpairs. Set $\beta_{0}=1, y_{0}=0$;

2. Apply wGKL to generate $X_{k}, Y_{k}, B_{k}, \beta_{k}$;

3. Compute an $S V D$ of $B_{k}$, select $\ell \leq k$ wanted singular values $\bar{\sigma}_{j}$ and their associated left and right singular vectors $\bar{\phi}_{j}$ and $\bar{\psi}_{j}$. Form $\pm \bar{\lambda}_{j}$ and $\bar{z}_{j}^{ \pm}$as in (2.5). Use (2.6) to verify convergence; if $\left\|\mathbf{H} \bar{z}_{j}^{+}-\bar{\lambda}_{j} \bar{z}_{j}^{+}\right\|_{\mathrm{M}} \leq$ tol, then stop.

The following lemma is critical to our later discussion.

Lemma 2.1 ([28, Prop. 3.1]). The matrix $M K$ has $n$ positive eigenvalues $\lambda_{1}^{2} \geq \lambda_{2}^{2} \geq$ $\cdots \geq \lambda_{n}^{2}>0$. The corresponding right eigenvectors $\xi_{1}, \ldots, \xi_{n}$ can be chosen $K$-orthonormal, and the corresponding left eigenvectors $\eta_{1}, \ldots, \eta_{n}$ can be chosen $M$-orthonormal. In particular, for given $\left\{\xi_{j}\right\}$, one can choose $\eta_{j}=\lambda_{j}^{-1} K \xi_{j}$, and for given $\left\{\eta_{j}\right\}, \xi_{j}=\lambda_{j}^{-1} M \eta_{j}$, for $j=1, \ldots, n$.

REMARK 2.2. From Lemma 2.1, it is not hard to obtain that $\lambda_{j}$ are the eigenvalues of $\mathbf{H}$ and $\left[\eta_{j}, \xi_{j}\right]^{T}$ are the corresponding eigenvectors. Moreover, we have that

$$
M K \Xi=\Xi \Lambda^{2}, \quad K M \Gamma=\Gamma \Lambda^{2},
$$

are the spectral decompositions of $M K$ and $K M$, respectively, where $\Xi=\left[\xi_{1}, \ldots, \xi_{n}\right]$, $\Gamma=\left[\eta_{1}, \ldots, \eta_{n}\right]$, and $\Lambda=\operatorname{diag}\left(\lambda_{1}, \ldots, \lambda_{n}\right)$.

REMARK 2.3. With $k$ increasing in Step 2, the $M$-orthogonality of $X_{k}$ and the $K$ orthogonality of $Y_{k}$ will slowly get lost. Thus, in practice, a reorthogonalization process can be added in each iteration to eliminate this shortcoming. The same strategy is executed in the following algorithms.

3. Weighted harmonic Golub-Kahan-Lanczos algorithm. In fact, the above algorithm WGKL-1 is a weighted Rayleigh-Ritz projection method. In this section, we first describe a weighted harmonic Golub-Kahan-Lanczos algorithm (WHGKL) in detail. Then we analyze the bound for the difference between the exact eigenvalue and the approximate one, together with their corresponding eigenvectors. Between wGKL-1 and wHGKL, we also discuss which one is better suited to solve exterior or interior eigenvalues for the LREP. Finally, the second type of a weighted Golub-Kahan-Lanczos algorithm (wGKL-2) is given, and the difference between WGKL-2 and wHGKL is illustrated. 
3.1. The principle of WHGKL. From Algorithm 1, we have equation (2.4). Making use of the harmonic projection condition, the weighted harmonic Golub-Kahan-Lanczos algorithm seeks the pair $\left(\hat{\lambda}_{j}, \hat{z}_{j}\right), j=1, \ldots, k$, which satisfies

$$
\left\{\begin{array}{l}
\hat{z}_{j} \in \operatorname{span}\left\{\mathbf{X}_{k}\right\}, \\
\mathbf{H} \hat{z}_{j}-\hat{\lambda}_{j} \hat{z}_{j} \perp_{\mathbf{M}} \mathbf{H} \operatorname{span}\left\{\mathbf{X}_{k}\right\},
\end{array}\right.
$$

where $\perp_{\mathbf{M}}$ denotes $\mathbf{M}$-orthogonality. The $\left(\hat{\lambda}_{j}, \hat{z}_{j}\right)$ is called the weighted harmonic Ritz pair of $\mathbf{H}$ in $\operatorname{span}\left\{\mathbf{X}_{k}\right\}$, and we let $\hat{z}_{j}=\left[\begin{array}{cc}X_{k} & 0 \\ 0 & Y_{k}\end{array}\right]\left[\begin{array}{c}g_{j} \\ f_{j}\end{array}\right]$. Thus from (3.1), we have

$$
\begin{aligned}
& {\left[\begin{array}{cc}
X_{k} & 0 \\
0 & Y_{k}
\end{array}\right]^{T}\left[\begin{array}{cc}
0 & K \\
M & 0
\end{array}\right]^{T}\left[\begin{array}{cc}
M & 0 \\
0 & K
\end{array}\right]\left[\begin{array}{cc}
0 & K \\
M & 0
\end{array}\right]\left[\begin{array}{cc}
X_{k} & 0 \\
0 & Y_{k}
\end{array}\right]\left[\begin{array}{l}
g_{j} \\
f_{j}
\end{array}\right]} \\
& =\hat{\lambda}_{j}\left[\begin{array}{cc}
X_{k} & 0 \\
0 & Y_{k}
\end{array}\right]^{T}\left[\begin{array}{cc}
0 & K \\
M & 0
\end{array}\right]^{T}\left[\begin{array}{cc}
M & 0 \\
0 & K
\end{array}\right]\left[\begin{array}{cc}
X_{k} & 0 \\
0 & Y_{k}
\end{array}\right]\left[\begin{array}{l}
g_{j} \\
f_{j}
\end{array}\right] .
\end{aligned}
$$

Rewriting the above equation, one has

$$
\left[\begin{array}{cc}
X_{k}^{T} M K M X_{k} & 0 \\
0 & Y_{k}^{T} K M K Y_{k}
\end{array}\right]\left[\begin{array}{l}
g_{j} \\
f_{j}
\end{array}\right]=\hat{\lambda}_{j}\left[\begin{array}{cc}
0 & X_{k}^{T} M K X_{k} \\
Y_{k}^{T} K M Y_{k} & 0
\end{array}\right]\left[\begin{array}{l}
g_{j} \\
f_{j}
\end{array}\right] .
$$

Recalling (2.2) and (2.3), equation (3.2) becomes

$$
\left[\begin{array}{cc}
B_{k}^{T} B_{k} & 0 \\
0 & B_{k} B_{k}^{T}+\beta_{k}^{2} e_{k} e_{k}^{T}
\end{array}\right]\left[\begin{array}{c}
g_{j} \\
f_{j}
\end{array}\right]=\hat{\lambda}_{j}\left[\begin{array}{cc}
0 & B_{k}^{T} \\
B_{k} & 0
\end{array}\right]\left[\begin{array}{c}
g_{j} \\
f_{j}
\end{array}\right]
$$

thus we have

$$
B_{k}^{T} B_{k} g_{j}=\hat{\lambda}_{j} B_{k}^{T} f_{j}, \quad\left(B_{k} B_{k}^{T}+\beta_{k}^{2} e_{k} e_{k}^{T}\right) f_{j}=\hat{\lambda}_{j} B_{k} g_{j} .
$$

REMARK 3.1. Actually, equation (3.4) looks similar to

$$
\left\{\begin{array}{l}
\tilde{\eta} B_{k, k} \tilde{d}=B_{k, k+1} B_{k, k+1}^{T} \tilde{c} \\
\tilde{\theta} B_{k, k}^{T} \tilde{c}=B_{k, k}^{T} B_{k, k} \tilde{d}
\end{array}\right.
$$

in [8, Sec. 7] except for the coefficients $\tilde{\eta}$ and $\tilde{\theta}$. Here we use the same coefficient $\hat{\lambda}$.

From the above, we know that $B_{k}^{T}=X_{k}^{T} M K X_{k}$ since $K$ and $M$ are positive definite and $X_{k}$ is $M$-orthonormal. Thus, $B_{k}^{T}$ is nonsingular. Left-multiplying the first equation of (3.4) by $B_{k}^{-T}$, we have $B_{k} g_{j}=\hat{\lambda}_{j} f_{j}$. We then substitute it into the second equation of (3.4) yielding

$$
B_{k, k+1} B_{k, k+1}^{T} f_{j}=\hat{\lambda}_{j}^{2} f_{j},
$$

where $B_{k, k+1}=\left[B_{k}, \beta_{k} e_{k}\right]$. Let the singular value decomposition of $B_{k, k+1}$ be

$$
B_{k, k+1}=\Phi_{k} \Sigma_{k} \Psi_{k+1, k}^{T},
$$

where $\Phi_{k} \in \mathbb{R}^{k \times k}, \Psi_{k+1, k} \in \mathbb{R}^{(k+1) \times k}, \Sigma_{k}=\operatorname{diag}\left(\sigma_{1}, \ldots, \sigma_{k}\right)$ satisfying $\Phi_{k}^{T} \Phi_{k}=I_{k}$, $\Psi_{k+1, k}^{T} \Psi_{k+1, k}=I_{k}, \sigma_{1} \geq \cdots \geq \sigma_{k}>0$. Consequently, $B_{k, k+1} B_{k, k+1}^{T}=\Phi_{k} \Sigma_{k}^{2} \Phi_{k}^{T}$. From equation (3.5), we can take $\hat{\lambda}_{j}=\sigma_{j}, f_{j}=\phi_{j}$, where $\phi_{j}$ is the $j$ th column of $\Phi_{k}$. Then 
$g_{j}=\hat{\lambda}_{j} B_{k}^{-1} f_{j}=\sigma_{j} B_{k}^{-1} \phi_{j}$. Note that all the eigenvalues of $\mathbf{H}$ are real and appear in pairs $\{\lambda,-\lambda\}$. Therefore, the approximate eigenpairs of $\mathbf{H}$ can be set as

$$
\pm \hat{\lambda}_{j}= \pm \sigma_{j}, \quad \hat{z}_{j}^{ \pm}=\left[\begin{array}{cc}
X_{k} & \\
& Y_{k}
\end{array}\right]\left[\begin{array}{c}
g_{j} \\
\pm f_{j}
\end{array}\right]=\left[\begin{array}{c}
\sigma_{j} X_{k} B_{k}^{-1} \phi_{j} \\
\pm Y_{k} \phi_{j}
\end{array}\right] .
$$

Next, we give the stopping criterion of WHGKL. Equation (3.3) can be written as

$$
\left[\begin{array}{cc}
B_{k}^{T} B_{k} & 0 \\
0 & B_{k} B_{k}^{T}
\end{array}\right]\left[\begin{array}{c}
g_{j} \\
f_{j}
\end{array}\right]+\left[\begin{array}{cc}
0 & 0 \\
0 & \beta_{k}^{2} e_{k} e_{k}^{T}
\end{array}\right]\left[\begin{array}{c}
g_{j} \\
f_{j}
\end{array}\right]=\hat{\lambda}_{j}\left[\begin{array}{cc}
0 & B_{k}^{T} \\
B_{k} & 0
\end{array}\right]\left[\begin{array}{c}
g_{j} \\
f_{j}
\end{array}\right] .
$$

Left-multiplying the above equation by $\left[\begin{array}{cc}0 & B_{k}^{T} \\ B_{k} & 0\end{array}\right]^{-1}$ and moving the terms, we have

$$
\begin{aligned}
{\left[\begin{array}{cc}
0 & B_{k}^{T} \\
B_{k} & 0
\end{array}\right]\left[\begin{array}{c}
g_{j} \\
f_{j}
\end{array}\right]-\hat{\lambda}_{j}\left[\begin{array}{c}
g_{j} \\
f_{j}
\end{array}\right] } & =-\left[\begin{array}{cc}
0 & \beta_{k}^{2} B_{k}^{-1} e_{k} e_{k}^{T} \\
0 & 0
\end{array}\right]\left[\begin{array}{c}
g_{j} \\
f_{j}
\end{array}\right] \\
& =-\beta_{k}^{2} e_{k}^{T} f_{j}\left[\begin{array}{c}
B_{k}^{-1} e_{k} \\
0
\end{array}\right] .
\end{aligned}
$$

It follows from (2.4) that we have

$$
\begin{aligned}
\mathbf{H} \hat{z}_{j}^{+}-\hat{\lambda}_{j} \hat{z}_{j}^{+} & =\left[\begin{array}{cc}
0 & K \\
M & 0
\end{array}\right]\left[\begin{array}{ll}
X_{k} & \\
& Y_{k}
\end{array}\right]\left[\begin{array}{c}
g_{j} \\
f_{j}
\end{array}\right]-\hat{\lambda}_{j}\left[\begin{array}{ll}
X_{k} & \\
& Y_{k}
\end{array}\right]\left[\begin{array}{c}
g_{j} \\
f_{j}
\end{array}\right] \\
& =\left[\begin{array}{ll}
X_{k} & \\
& Y_{k}
\end{array}\right]\left(\left[\begin{array}{cc}
0 & B_{k}^{T} \\
B_{k} & 0
\end{array}\right]\left[\begin{array}{c}
g_{j} \\
f_{j}
\end{array}\right]-\hat{\lambda}_{j}\left[\begin{array}{c}
g_{j} \\
f_{j}
\end{array}\right]\right)+\beta_{k} e_{k}^{T} f_{j}\left[\begin{array}{c}
x_{k+1} \\
0
\end{array}\right] \\
& =\beta_{k} e_{k}^{T} f_{j}\left[\begin{array}{cc}
X_{k+1} & \\
& Y_{k}
\end{array}\right]\left[\begin{array}{c}
-\beta_{k} B_{k}^{-1} e_{k} \\
1 \\
0
\end{array}\right]
\end{aligned}
$$

So we can use

$$
\left\|\mathbf{H} \hat{z}_{j}^{+}-\hat{\lambda}_{j} \hat{z}_{j}^{+}\right\|_{\mathbf{M}}=\beta_{k}^{2}\left|f_{j k}\right| \sqrt{\frac{1}{\beta_{k}^{2}}+\left\|B^{-1} e_{k}\right\|_{2}^{2}}
$$

as the stopping criterion, where $f_{j k}$ is the $k$ th element of $f_{j}$. Thus we have the following algorithm.

Algorithm 3 (whGKL).

1. Given an initial guess $x_{1}$ satisfying $\left\|x_{1}\right\|_{M}=1$, a tolerance tol, an integer $m$ the dimension of the solution subspace, as well as $\ell$ the desired number of eigenpairs. Set $\beta_{0}=1, y_{0}=0$;

2. Apply WGKL to generate $X_{k}, Y_{k}, B_{k, k+1}$;

3. Compute an SVD of $B_{k, k+1}$ as in (3.6), select $\ell \leq k$ wanted singular values $\sigma_{j}$ and their associated left singular vectors $\phi_{j}$. Form $\pm \hat{\lambda}_{j}$ and $\hat{z}_{j}^{ \pm}$as in (3.7). Use (3.8) to verify convergence; if $\left\|\mathbf{H} \hat{z}_{j}^{+}-\hat{\lambda}_{j} \hat{z}_{j}^{+}\right\|_{\mathbf{M}} \leq$ tol, then stop.

3.2. Convergence analysis. Naturally, the few largest $\sigma_{j}$ are used to approximate the few largest $\lambda_{j}$, and the few smallest $\sigma_{j}$ are used to approximate the few smallest $\lambda_{j}$. In this section, we give the accuracy of these approximates. Firstly, the bounds for the errors in $\sigma_{j}^{2}$ as an approximation to $\lambda_{j}^{2}$, together with their corresponding eigenvector, are presented in the following theorem. We denote by $C_{j}(x)$ the Chebyshev polynomial of the first kind of degree $j$ [21], and two angles are defined as follows:

$$
\theta(x, y)=\arccos \frac{\left|x^{T} y\right|}{\|x\|\|y\|}, \quad \theta_{A}(x, y)=\arccos \frac{\left|(x, y)_{A}\right|}{\|x\|_{A}\|y\|_{A}},
$$


where $0 \neq x, y \in \mathbb{R}^{n}, 0<A \in \mathbb{R}^{n \times n}$.

THEOREM 3.2 (cf. [28, Thm. 3.2]). For $j=1,2, \ldots, n$, suppose that the eigenvalues of $M K$ are $\lambda_{1}^{2} \geq \cdots \geq \lambda_{n}^{2}>0$ and $\xi_{1}, \ldots, \xi_{n}$ are their corresponding $K$-orthogonal eigenvectors. Suppose that Algorithm 1 does not break down at the kth iteration. Let the SVD of $B_{k, k+1}$ be (3.6), and $\sigma_{1} \geq \cdots \geq \sigma_{k}>0$, and let $w=\Xi^{T} K M x_{1}=\left[w_{1}, \ldots, w_{n}\right]^{T}$. Then, for $j=1, \ldots, k$, there holds

$$
0 \leq \lambda_{j}^{2}-\sigma_{j}^{2} \leq\left(\lambda_{j}^{2}-\lambda_{n}^{2}\right)\left(\frac{\pi_{j, k} \mu_{j}}{C_{k-j}\left(1+2 \gamma_{j}\right)}\right)^{2}
$$

and

$$
\begin{aligned}
& \sqrt{\lambda_{j}^{2}\left\|B_{k}^{-1} \phi_{j}\right\|_{2}^{2} \sin ^{2} \theta_{M}\left(\sigma_{j} X_{k} B_{k}^{-1} \phi_{j}, \eta_{j}\right)+1-\lambda_{j}^{2}\left\|B_{k}^{-1} \phi_{j}\right\|_{2}^{2}} \\
& =\sin \theta_{K}\left(Y_{k} \phi_{j}, \xi_{j}\right) \leq \frac{\pi_{j} \sqrt{1+\left(\alpha_{k+1} \beta_{k}\right)^{2} / \delta_{j}^{2}}}{C_{k-j}\left(1+2 \gamma_{j}\right)} \tan \theta_{K}\left(M x_{1}, \xi_{j}\right)
\end{aligned}
$$

where

$$
\begin{aligned}
\pi_{j, k} & =\max _{j+1 \leq t \leq n} \prod_{i=1}^{j-1}\left|\frac{\sigma_{i}^{2}-\lambda_{t}^{2}}{\sigma_{i}^{2}-\lambda_{j}^{2}}\right|, \quad \mu_{j}=\frac{\sqrt{\sum_{i=j+1}^{n}\left|w_{i}\right|^{2}}}{\left|w_{j}\right|}, \quad \gamma_{j}=\frac{\lambda_{j}^{2}-\lambda_{j+1}^{2}}{\lambda_{j+1}^{2}-\lambda_{n}^{2}}, \\
\pi_{j} & =\prod_{i=1}^{j-1} \frac{\lambda_{i}^{2}-\lambda_{n}^{2}}{\lambda_{i}^{2}-\lambda_{j}^{2}}, \quad \delta_{j}=\min _{i \neq j}\left|\lambda_{j}^{2}-\sigma_{i}^{2}\right| .
\end{aligned}
$$

Proof. Since $K>0, K$ has a factorization $K=L L^{T}$ with $L$ invertible. From Lemma 2.1 and (2.7), it is easy to see that for any $j=1, \ldots, n, \lambda_{j}^{2}$ is an eigenvalue of $L^{T} M L$ with the corresponding eigenvector $L^{T} \xi_{j}$, i.e.,

$$
L^{T} M L=L^{T} \Xi \Lambda^{2}\left(L^{T} \Xi\right)^{T}
$$

is the spectral decomposition of $L^{T} M L$ and $L^{T} \xi_{1}, \ldots, L^{T} \xi_{n}$ are orthonormal.

Left-multiplying the second equation of (2.2) by $M$, we get

$$
M K Y_{k}=Y_{k} B_{k, k+1} B_{k, k+1}^{T}+\alpha_{k+1} \beta_{k} y_{k+1} e_{k}^{T} .
$$

Let $A=L^{T} M L, T_{k}=B_{k, k+1} B_{k, k+1}^{T}$, and transform the above equation to

$$
A V_{k}=V_{k} T_{k}+\alpha_{k+1} \beta_{k} v_{k+1} e_{k}^{T}
$$

with $V_{k}=L^{T} Y_{k}$ and $v_{k}=L^{T} y_{k}$. Obviously, $V_{k+1}$ is orthonormal, and (3.13) can be considered as the relation obtained by using the standard Lanczos algorithm for $A$. Then for all $u \in \mathbb{R}^{k}$, there exists $\chi \in \mathbb{P}_{k-1}$ such that $V_{k} u=\chi(A) v_{1}$, where $\mathbb{P}_{k-1}$ is the set of all polynomials of degree less than or equal to $k-1$ with coefficients in $\mathbb{R}$. From the SVD of $B_{k, k+1}$ (3.6), we can also see that $\sigma_{1}^{2}, \ldots, \sigma_{k}^{2}$ are the Ritz values of $A, V_{k} \phi_{1}, \ldots, V_{k} \phi_{k}$ are the corresponding orthonormal Ritz vectors, and $T_{k}=\Phi_{k} \Sigma_{k}^{2} \Phi_{k}^{T}$ is the spectral decomposition of $T_{k}$.

From [28], we know that if Algorithm 1 does not break down at the $k$ th iteration, then $\alpha_{j}, \beta_{j} \neq 0, j=1, \ldots, k$. Hence, $T_{k}$ is an irreducible symmetric tridiagonal matrix, so for 
any $u \in \mathbb{R}^{k}, u \perp \phi_{i}$ means that $\chi\left(\sigma_{i}^{2}\right)=0, i=1, \ldots, j-1$. Note that $w=\Xi^{T} K M x_{1}=$ $\alpha_{1}\left(L^{T} \Xi\right)^{T} v_{1}$ by Algorithm 1. Then we have

$$
\begin{aligned}
\sigma_{j}^{2} & =\max _{\substack{u \perp \phi_{i} \\
1 \leq i \leq j-1}} \frac{u^{T} T_{k} u}{u^{T} u}=\max _{\substack{\left.\chi \sigma_{i}^{2}\right)=0 \\
1 \leq i \leq j-1}} \frac{u^{T} V_{k}^{T} A V_{k} u}{u^{T} V_{k}^{T} V_{k} u} \\
& =\max _{\substack{\chi\left(\sigma_{i}^{2}\right)=0 \\
1 \leq i \leq j-1}} \frac{v_{1}^{T} \chi(A) A \chi(A) v_{1}}{v_{1}^{T} \chi^{2}(A) v_{1}} \\
& =\max _{\substack{\chi\left(\sigma_{i}^{2}\right)=0 \\
1 \leq i \leq j-1}} \frac{v_{1}^{T} L^{T} \Xi \chi\left(\Lambda^{2}\right) \Lambda^{2} \chi\left(\Lambda^{2}\right)\left(L^{T} \Xi\right)^{T} v_{1}}{v_{1}^{T} L^{T} \Xi \chi^{2}\left(\Lambda^{2}\right)\left(L^{T} \Xi\right)^{T} v_{1}} \\
& =\max _{\substack{\chi\left(\sigma_{i}^{2}\right)=0 \\
1 \leq i \leq j-1}} \frac{w^{T} \chi\left(\Lambda^{2}\right) \Lambda^{2} \chi\left(\Lambda^{2}\right) w}{w^{T} \chi^{2}\left(\Lambda^{2}\right) w},
\end{aligned}
$$

where the last equality is using the spectral decomposition (3.11) of $A$. Again using (3.11) and (3.13), we have $\lambda_{j} \geq \sigma_{j} \geq \lambda_{n-k+j}$, which is a consequence of the Cauchy interlacing inequalities applied to $A$ and $T_{k}, j=1, \ldots, k$. Thus,

$$
\begin{aligned}
0 \leq \lambda_{j}^{2}-\sigma_{j}^{2} & =\min _{\substack{\chi\left(\sigma_{i}^{2}\right)=0 \\
1 \leq i \leq j-1}} \frac{w^{T} \chi\left(\Lambda^{2}\right)\left(\lambda_{j}^{2}-\Lambda^{2}\right) \chi\left(\Lambda^{2}\right) w}{w^{T} \chi^{2}\left(\Lambda^{2}\right) w} \\
& =\min _{\substack{\chi\left(\sigma_{i}^{2}\right)=0 \\
1 \leq i \leq j-1}} \frac{\sum_{i=1}^{n}\left(\lambda_{j}^{2}-\lambda_{i}^{2}\right) \chi^{2}\left(\lambda_{i}^{2}\right)\left|w_{i}\right|^{2}}{\sum_{i=1}^{n} \chi^{2}\left(\lambda_{i}^{2}\right)\left|w_{i}\right|^{2}} \\
& \leq \min _{\substack{\chi\left(\sigma_{i}^{2}\right)=0 \\
1 \leq i \leq j-1}} \frac{\sum_{i=j+1}^{n}\left(\lambda_{j}^{2}-\lambda_{i}^{2}\right) \chi^{2}\left(\lambda_{i}^{2}\right)\left|w_{i}\right|^{2}}{\chi^{2}\left(\lambda_{j}^{2}\right)\left|w_{j}\right|^{2}} .
\end{aligned}
$$

Take

$$
\chi(t)=\left(t-\sigma_{1}^{2}\right) \cdots\left(t-\sigma_{j-1}^{2}\right) C_{k-j}(\tau), \quad \text { with } \quad \tau=1+2 \frac{t-\lambda_{j+1}^{2}}{\lambda_{j+1}^{2}-\lambda_{n}^{2}} .
$$

It is easy to verify that $-1 \leq \tau \leq 1$ for $\lambda_{n}^{2} \leq t \leq \lambda_{j+1}^{2}$, and

$$
\text { (3.16) }\left.\tau\right|_{t=\lambda_{j}^{2}}=1+2 \gamma_{j}, \quad\left|\chi\left(\lambda_{i}^{2}\right)\right| \leq \max _{j+1 \leq t \leq n} \prod_{i=j+1}^{n}\left|\lambda_{t}^{2}-\sigma_{i}^{2}\right|, \quad \text { for } j+1 \leq i \leq n \text {. }
$$

Thus, the bound (3.9) is now a consequence of (3.14)-(3.16).

Next, let us turn to proving (3.10). Similarly to the proof of Theorem 3.2 in [28], applying the standard Lanczos convergence results in [18, Sec. 6.6] to (3.13), one has

$$
\sin \theta\left(V_{k} \phi_{j}, L^{T} \xi_{j}\right) \leq \frac{\pi_{j} \sqrt{1+\left(\alpha_{k+1} \beta_{k}\right)^{2} / \delta_{j}^{2}}}{C_{k-j}\left(1+2 \gamma_{j}\right)} \tan \theta\left(L^{T} y_{1}, L^{T} \xi_{j}\right) .
$$

Hence, the bound (3.10) can be obtained by using the identities

$$
\theta\left(L^{T} y_{1}, L^{T} \xi_{j}\right)=\theta_{K}\left(y_{1}, \xi_{j}\right)=\theta_{K}\left(M x_{1}, \xi_{j}\right), \quad \theta\left(V_{k} \phi_{j}, L^{T} \xi_{j}\right)=\theta_{K}\left(Y_{k} \phi_{j}, \xi_{j}\right) .
$$


Finally, we prove the equality in (3.10). By the first equation of (2.2), we know that $Y_{k} \phi_{j}=\frac{1}{\sigma_{j}} \sigma_{j} M X_{k} B_{k}^{-1} \phi_{j}$. Then from Lemma 2.1, one obtains

$$
\begin{aligned}
\sin ^{2} \theta_{K}\left(Y_{k} \phi_{j}, \xi_{j}\right) & =1-\cos ^{2} \theta_{K}\left(Y_{k} \phi_{k}, \xi_{j}\right)=1-\left|\left(Y_{k} \phi_{j}\right)^{T} K \xi_{j}\right|^{2} \\
& =1-\frac{\lambda_{j}^{2}}{\sigma_{j}^{2}}\left|\left(\sigma_{j} M X_{k} B_{k}^{-1} \phi_{j}\right)^{T} \frac{K \xi_{j}}{\lambda_{j}}\right|^{2} \\
& =1-\frac{\lambda_{j}^{2}}{\sigma_{j}^{2}}\left\|\sigma_{j} X_{k} B_{k}^{-1} \phi_{j}\right\|_{M}^{2} \frac{\left|\left(\sigma_{j} X_{k} B_{k}^{-1} \phi_{j}\right)^{T} M \eta_{j}\right|^{2}}{\left\|\sigma_{j} X_{k} B_{k}^{-1} \phi_{j}\right\|_{M}^{2}} \\
& =1-\lambda_{j}^{2}\left\|B_{k}^{-1} \phi_{j}\right\|_{2}^{2} \cos ^{2} \theta_{M}\left(\sigma_{j} X_{k} B_{k}^{-1} \phi_{j}, \eta_{j}\right) \\
& =1-\lambda_{j}^{2}\left\|B_{k}^{-1} \phi_{j}\right\|_{2}^{2}+\lambda_{j}^{2}\left\|B_{k}^{-1} \phi_{j}\right\|_{2}^{2} \sin ^{2} \theta_{M}\left(\sigma_{j} X_{k} B_{k}^{-1} \phi_{j}, \eta_{j}\right) .
\end{aligned}
$$

Taking the square root of the above equality completes the proof.

REMARK 3.3. Because WHGKL differs from WGKL-1, we have presented Theorem 3.2 to prove convergence for WHGKL similar to equation (3.6) in [28, Theorem 3.2], which is the convergence proof of WGKL-1. Moreover, the proofs of the two theorems are different. In [28], the standard Lanczos convergence results in [18, Sec. 6.6] are applied to (3.13), while here, we use the min-max principle [20, Thm. 3.5] for the matrix $T_{k}$.

REMARK 3.4. Since $B_{k, k+1}=\left[B_{k}, \beta_{k} e_{k}\right]$, if the difference between the SVD of $B_{k, k+1}$ and $B_{k}$ is tiny, then $\left\|B_{k}^{-1} \phi_{j}\right\|_{2}$ is very close to $\sigma_{j}^{-1}$. Consequently, from the left part of (3.10), we can see that when $\sigma_{j}$ is close to $\lambda_{j}$, the accuracy of the approximation $Y_{k} \phi_{j}$ of $\xi_{j}$ is related to the accuracy of the approximation $\sigma_{j} X_{k} B_{k}^{-1} \phi_{j}$ of $\eta_{j}$.

REMARK 3.5. Theorem 3.2 and the following Theorems 3.6, 3.7 bound the angles between $Y_{k} \phi_{j}$ and the eigenvector $\xi_{j}$ or $\xi_{n-k+j}$ of $M K$ for WHGKL and WGKL-2 (as is discussed in Section 3.3). The values of $C_{k-j}\left(1+2 \gamma_{j}\right)$ and $C_{k-j}\left(1+2 \bar{\gamma}_{j}\right)$ are fixed for fixed $j$, and they will not be less than 1 . The values of $\tan \theta_{K}\left(M x_{1}, \xi_{j}\right)$ and $\tan \theta_{K}\left(M x_{1}, \xi_{n-k+j}\right)$ represent the effect of the initial vector $x_{1}$ to the approximated eigenvectors.

Similar results can be obtained for the errors in $\sigma_{j}^{2}$ as an approximation to $\lambda_{n-k+j}^{2}$.

THEOREM 3.6. With the same conditions and notations as in Theorem 3.2, then for $j=k, k-1, \ldots, 1$, there holds

$$
0 \leq \sigma_{j}^{2}-\lambda_{n-k+j}^{2} \leq\left(\lambda_{1}^{2}-\lambda_{n-k+j}^{2}\right)\left(\frac{\bar{\pi}_{j, k} \bar{\mu}_{j}}{C_{k-j}\left(1+2 \bar{\gamma}_{j}\right)}\right)^{2}
$$

and

$$
\begin{gathered}
\sqrt{\lambda_{n-k+j}^{2}\left\|B_{k}^{-1} \phi_{j}\right\|_{2}^{2} \sin ^{2} \theta_{M}\left(\sigma_{j} X_{k} B_{k}^{-1} \phi_{j}, \eta_{n-k+j}\right)+1-\lambda_{n-k+j}^{2}\left\|B_{k}^{-1} \phi_{j}\right\|_{2}^{2}} \\
=\sin \theta_{K}\left(Y_{k} \phi_{j}, \xi_{n-k+j}\right) \leq \frac{\bar{\pi}_{j} \sqrt{1+\left(\alpha_{k+1} \beta_{k}\right)^{2} / \bar{\delta}_{j}^{2}}}{C_{k-j}\left(1+2 \bar{\gamma}_{j}\right)} \tan \theta_{K}\left(M x_{1}, \xi_{n-k+j}\right),
\end{gathered}
$$

where

$$
\begin{gathered}
\bar{\pi}_{j, k}=\max _{1 \leq t \leq n-k+j-1} \prod_{i=j+1}^{k}\left|\frac{\sigma_{i}^{2}-\lambda_{t}^{2}}{\sigma_{i}^{2}-\lambda_{n-k+j}^{2}}\right|, \quad \bar{\mu}_{j}=\frac{\sqrt{\sum_{i=1}^{n-k+j-1}\left|w_{i}\right|^{2}}}{\left|w_{n-k+j}\right|}, \\
\bar{\gamma}_{j}=\frac{\lambda_{n-k+j-1}^{2}-\lambda_{n-k+j}^{2}}{\lambda_{1}^{2}-\lambda_{n-k+j-1}^{2}}, \quad \bar{\pi}_{j}=\prod_{i=n-k+j+1}^{n} \frac{\lambda_{i}^{2}-\lambda_{1}^{2}}{\lambda_{i}^{2}-\lambda_{n-k+j}^{2}}, \quad \bar{\delta}_{j}=\min _{i \neq j}\left|\lambda_{n-k+j}^{2}-\sigma_{i}^{2}\right| .
\end{gathered}
$$


3.3. The second weighted Golub-Kahan-Lanczos algorithm. In this section, we describe another way to approximate eigenpairs of $\mathbf{H}$ by using $B_{k, k+1}$, i.e., the second weighted Golub-Kahan-Lanczos algorithm, denoted by WGKL-2. In addition, we provide its new convergence theory and analyze the difference between WGKL-2 and WHGKL.

Suppose that $y_{k+1}$ is computed by wGKL but $x_{k+2}$ is not computed. Then the following relations hold:

$$
\begin{aligned}
K Y_{k}=X_{k+1} B_{k, k+1}^{T}, \quad M X_{k+1} & =Y_{k} B_{k, k+1}+\alpha_{k+1} y_{k+1} e_{k+1}^{T} \\
X_{k+1}^{T} M X_{k+1} & =I_{k+1}=Y_{k+1}^{T} K Y_{k+1}
\end{aligned}
$$

Define

$$
\overline{\mathbf{X}}_{k}=\left[\begin{array}{ll}
X_{k+1} & \\
& Y_{k}
\end{array}\right], \quad \overline{\mathbf{B}}_{k}=\left[\begin{array}{ll}
B_{k, k+1}^{T} &
\end{array}\right]
$$

Then

$$
\mathbf{H} \overline{\mathbf{X}}_{k}=\overline{\mathbf{X}}_{k} \overline{\mathbf{B}}_{k}+\alpha_{k+1}\left[\begin{array}{c}
0 \\
y_{k+1}
\end{array}\right] e_{2 k+1}^{T} .
$$

Hence, from the SVD of $B_{k, k+1}$ (3.6) and (3.18), we can take

$$
\pm \tilde{\lambda}_{j}= \pm \sigma_{j}, \quad \tilde{z}_{j}^{ \pm}=\frac{1}{\sqrt{2}}\left[\begin{array}{c}
X_{k+1} \psi_{j} \\
\pm Y_{k} \phi_{j}
\end{array}\right], \quad j=1, \ldots, k
$$

as the Ritz values and the corresponding $\mathbf{M}$-orthonormal Ritz vectors of $\mathbf{H}$. We may take

$$
\left\|\mathbf{H} \tilde{z}_{j}^{+}-\tilde{\lambda}_{j} \tilde{z}_{j}^{+}\right\|_{\mathbf{M}}=\frac{\alpha_{k+1}\left|\phi_{j k}\right|}{\sqrt{2}}
$$

as the stopping criteria. Here $\phi_{j k}$ is the $k$-th element of $\phi_{j}$. The following algorithm is the second weighted Golub-Kahan-Lanczos algorithm (wGKL-2).

Algorithm 4 (wGKL-2).

1. Given an initial guess $x_{1}$ satisfying $\left\|x_{1}\right\|_{M}=1$, a tolerance tol, an integer $m$ (the dimension of the solution subspace) as well as $\ell$ (the desired number of eigenpairs). Set $\beta_{0}=1, y_{0}=0$;

2. Apply wGKL to generate $X_{k+1}, Y_{k}, B_{k, k+1}, \alpha_{k+1}$;

3. Compute an SVD of $B_{k, k+1}$ as in (3.6), select $\ell \leq k$ wanted singular values $\sigma_{j}$ and their associated left and right singular vectors $\phi_{j}$ and $\psi_{j}$. Form $\pm \hat{\lambda}_{j}$ and $\hat{z}_{j}^{ \pm}$as in (3.19). Use (3.20) to verify convergence; if $\left\|\mathbf{H} \tilde{z}_{j}^{+}-\tilde{\lambda}_{j} \tilde{z}_{j}^{+}\right\|_{\mathrm{M}} \leq$ tol, then stop.

Since left-multiplying the first equation in (3.17) by $M$ yields (3.12), we can establish the convergence theory of wGKL-2 similar to WHGKL. Theorem 3.7 provides the bounds for the errors in $\sigma_{j}^{2}$ as an approximation to $\lambda_{j}^{2}$ together with their corresponding eigenvector and also gives the parallel results applied to the errors in $\sigma_{j}^{2}$ as an approximation for $\lambda_{n-k+j}^{2}$.

THEOREM 3.7. With the same conditions and notations as in Theorem 3.2 and 3.6, for $j=1, \ldots, k$, there holds

$$
0 \leq \lambda_{j}^{2}-\sigma_{j}^{2} \leq\left(\lambda_{j}^{2}-\lambda_{n}^{2}\right)\left(\frac{\pi_{j, k} \mu_{j}}{C_{k-j}\left(1+2 \gamma_{j}\right)}\right)^{2}
$$


and

$$
\begin{aligned}
& \sqrt{\frac{\sigma_{j}^{2}}{\lambda_{j}^{2}} \sin ^{2} \theta_{M}\left(X_{k+1} \psi_{j}, \eta_{j}\right)+1-\frac{\sigma_{j}^{2}}{\lambda_{j}^{2}}} \\
& =\sin \theta_{K}\left(Y_{k} \phi_{j}, \xi_{j}\right) \leq \frac{\pi_{j} \sqrt{1+\left(\alpha_{k+1} \beta_{k}\right)^{2} / \delta_{j}^{2}}}{C_{k-j}\left(1+2 \gamma_{j}\right)} \tan \theta_{K}\left(M x_{1}, \xi_{j}\right) .
\end{aligned}
$$

Moreover, for $j=k, k-1, \ldots, 1$, there holds

$$
0 \leq \sigma_{j}^{2}-\lambda_{n-k+j}^{2} \leq\left(\lambda_{1}^{2}-\lambda_{n-k+j}^{2}\right)\left(\frac{\bar{\pi}_{j, k} \bar{\mu}_{j}}{C_{k-j}\left(1+2 \bar{\gamma}_{j}\right)}\right)^{2}
$$

and

$$
\begin{aligned}
& \sqrt{\frac{\sigma_{j}^{2}}{\lambda_{n-k+j}^{2}} \sin ^{2} \theta_{M}\left(X_{k+1} \psi_{j}, \eta_{n-k+j}\right)+1-\frac{\sigma_{j}^{2}}{\lambda_{n-k+j}^{2}}} \\
& =\sin \theta_{K}\left(Y_{k} \phi_{j}, \xi_{n-k+j}\right) \leq \frac{\bar{\pi}_{j} \sqrt{1+\left(\alpha_{k+1} \beta_{k}\right)^{2} / \bar{\delta}_{j}^{2}}}{C_{k-j}\left(1+2 \bar{\gamma}_{j}\right)} \tan \theta_{K}\left(M x_{1}, \xi_{n-k+j}\right) .
\end{aligned}
$$

REMARK 3.8. By observing the upper bound of $\lambda_{j}^{2}-\sigma_{j}^{2}$ in Theorem 3.7, we can easily find that

$$
\lambda_{j}^{2}-\lambda_{n}^{2} \leq \lambda_{1}^{2}-\lambda_{n}^{2}, \quad \mu_{j}=\frac{\sqrt{\sum_{i=j+1}^{n}\left|w_{i}\right|^{2}}}{\left|w_{j}\right|} \leq \frac{\sqrt{\sum_{j \neq i=1}^{n}\left|w_{i}\right|^{2}}}{\left|w_{j}\right|}=\tan \theta_{K}\left(M x_{1}, \xi_{j}\right) .
$$

This means that the bound here is tighter than the bound of $\lambda_{j}^{2}-\sigma_{j}^{2}$ in [28, Thm. 3.3], and the same results for $\sigma_{j}^{2}-\lambda_{n-k+j}^{2}$ hold.

REMARK 3.9. From the above discussion, we can see that WGKL-1 uses the singular values $\bar{\sigma}_{j}$ of $B_{k}$ as the approximate eigenvalues of $\mathbf{H}$, while WHGKL and WGKL-2 use the singular values $\sigma_{j}$ of $B_{k, k+1}$. Similar to Remark 3.4 in [28], because $\bar{\sigma}_{1} \geq \cdots \geq \bar{\sigma}_{k}$ are the singular value of $B_{k}$ and $\sigma_{1} \geq \cdots \geq \sigma_{k}$ are the singular value of $B_{k, k+1}$, using the interlacing properties [7, Cor. 8.6.3], we have

$$
\sigma_{1} \geq \bar{\sigma}_{1} \geq \cdots \geq \sigma_{k} \geq \bar{\sigma}_{k}
$$

Hence, from Theorems 3.2, 3.6, and 3.7, $\sigma_{j}$ will be more accurate for approximating a large eigenvalue $\lambda_{j}$ of $\mathbf{H}$, and $\bar{\sigma}_{j}$ will be more accurate for approximating a small eigenvalue $\lambda_{j}$ of $\mathbf{H}$.

REMARK 3.10. By comparing WGKL-2 and WHGKL, we can see that WGKL-2 needs $X_{k+1}, Y_{k}, B_{k, k+1}$, and $\alpha_{k+1}$ to form the approximated eigenpairs, while wHGKL needs $X_{k}$, $Y_{k}, B_{k, k+1}$, and $B_{k}^{-1}$. Thus, although WHGKL requires less computational effort with respect to matrix-vector products than wGKL-2, it needs much more computational effort for the inversion $B_{k}^{-1}$. Fortunately, $B_{k}$ is not only upper bidiagonal but also small. So the costs of this will generally be very modest compared to a matrix-vector product, and we use a direct solve with MATLAB backslash. 
4. Restart. With the size of the search space increasing, the storage and the computational costs of WHGKL grows quickly, and the $M$-orthogonality and $K$-orthogonality will be gradually lost, so its numerical stability will deteriorate. To overcome the difficulty, motivated by the idea in [2], we consider a thick-restarting procedure for WHGKL, i.e., we add some harmonic Ritz vectors to the search subspace for the purpose of acceleration. In fact, the methods in [2] can be seen as solving eigenproblems for a symmetric matrix $\left[\begin{array}{cc}0 & A^{T} \\ A & 0\end{array}\right]$, while in this paper, our matrix is $\left[\begin{array}{cc}0 & K \\ M & 0\end{array}\right]$, which is not symmetric. Therefore, we study how to apply their strategy to our problem. Details are discussed in the following.

Assume that WHGKL runs $m$ steps and generates the matrices $X_{m+1}, Y_{m}, B_{m}$, and $B_{m, m+1}=\left[B_{m}, \beta_{m} e_{m}\right]$. Let $B_{m, m+1}=\Phi_{m} \Sigma_{m} \Psi_{m+1, m}^{T}$ be the SVD of $B_{m, m+1}$, and let $\Phi_{m, s}$ be the first $s$ columns of $\Phi_{m}$. Here, the integer $s \leq m$ is the number of added harmonic Ritz vectors. Take the $\mathrm{QR}$ decomposition,

$$
\left[\begin{array}{cc}
B_{m}^{-1} \Phi_{m, s} \Sigma_{s} & -\beta_{m} B_{m}^{-1} e_{m} \\
0 & 1
\end{array}\right]=Q_{s+1} R_{s+1}
$$

where $Q_{s+1} \in \mathbb{R}^{(s+1) \times(s+1)}$ is orthogonal and $R_{s+1} \in \mathbb{R}^{(s+1) \times(s+1)}$ is an upper tridiagonal matrix, whose $(s+1) \times(s+1)$ element is 1 .

Let

$$
\widetilde{X}_{s+1}=\left[\tilde{x}_{1}, \ldots, \tilde{x}_{s+1}\right]=\left[X_{m}, x_{m+1}\right] Q_{s+1} .
$$

Then $\widetilde{X}_{s+1}^{T} M \widetilde{X}_{s+1}=I_{s+1}$, and

$$
\begin{aligned}
M \widetilde{X}_{s+1} & =\left[M X_{m}, M x_{m+1}\right]\left[\begin{array}{cc}
B_{m}^{-1} \Phi_{m, s} \Sigma_{s} & -\beta_{m} B_{m}^{-1} e_{m} \\
0 & 1
\end{array}\right] R_{s+1}^{-1} \\
& =\left[Y_{m} B_{m}, M x_{m+1}\right]\left[\begin{array}{cc}
B_{m}^{-1} \Phi_{m, s} \Sigma_{k} & -\beta_{m} B_{m}^{-1} e_{m} \\
0 & 1
\end{array}\right] R_{s+1}^{-1} \\
& =\left[Y_{m} \Phi_{m, s} \Sigma_{s},-\beta_{m} y_{m}+M x_{m+1}\right] R_{s+1}^{-1} .
\end{aligned}
$$

Now let

$$
\widetilde{Y}_{s}=Y_{m} \Phi_{m, s}
$$

and $\widetilde{Y}_{k}^{T} K\left(-\beta_{m} y_{m}+M x_{m+1}\right)=\left[\tilde{\gamma}_{1}, \ldots, \tilde{\gamma}_{s}\right]^{T}=\widetilde{C}_{s}$, and let

$$
-\beta_{m} y_{m}+M x_{m+1}-\widetilde{Y}_{s} \widetilde{C}_{s}=\tilde{\alpha}_{s+1} \tilde{y}_{s+1}
$$

where $\tilde{\alpha}_{s+1}$ is the value such that $\left\|\tilde{y}_{s+1}\right\|_{K}=1$. Thus $\tilde{Y}_{s+1}^{T} K \tilde{Y}_{s+1}=I_{s+1}$, and

$$
M \widetilde{X}_{s+1}=\left[\widetilde{Y}_{s}, \tilde{y}_{s+1}\right]\left[\begin{array}{cccc}
\sigma_{1} & & & \tilde{\gamma}_{1} \\
& \ddots & & \vdots \\
& & \sigma_{s} & \tilde{\gamma}_{s} \\
0 & \cdots & 0 & \tilde{\alpha}_{s+1}
\end{array}\right] R_{s+1}^{-1}=\widetilde{Y}_{s+1} \widetilde{B}_{s+1}
$$

where $\tilde{Y}_{s+1}=\left[\tilde{Y}_{s}, \tilde{y}_{s+1}\right]$ and

$$
\widetilde{B}_{s+1}=\left[\begin{array}{cccc}
\sigma_{1} & & & \tilde{\gamma}_{1} \\
& \ddots & & \vdots \\
& & \sigma_{s} & \tilde{\gamma}_{s} \\
0 & \cdots & 0 & \tilde{\alpha}_{s+1}
\end{array}\right] R_{s+1}^{-1}
$$


Obviously, the $(s+1, s+1)$ element of $\widetilde{B}_{s+1}$ is $\tilde{\alpha}_{s+1}$.

Since the SVD of $B_{m, m+1}$ is $B_{m, m+1}=\Phi_{m} \Sigma_{m} \Psi_{m+1, m}^{T}$, we have

$$
B_{m, m+1}^{T} \Phi_{m, s}=\Psi_{m+1, s} \Sigma_{s}
$$

and

$$
\left[B_{m}, \beta_{m} e_{m}\right] \Psi_{m+1, s}=\Phi_{m, s} \Sigma_{s}
$$

From (4.8), we get $\left[I_{m}, \beta_{m} B_{m}^{-1} e_{m}\right] \Psi_{m+1, k}=B_{m}^{-1} \Phi_{m, s} \Sigma_{s}$. Thus,

$$
\begin{aligned}
\Psi_{m+1, s} & =\left[\begin{array}{cc}
B_{m}^{-1} \Phi_{m, s} \Sigma_{s} & -\beta_{m} B_{m}^{-1} e_{m} \\
0 & 1
\end{array}\right]\left[\begin{array}{c}
I_{s} \\
e_{m+1}^{T} \Psi_{m+1, s}
\end{array}\right] \\
& =Q_{s+1} R_{s+1}\left[\begin{array}{c}
I_{s} \\
e_{m+1}^{T} \Psi_{m+1, s}
\end{array}\right] .
\end{aligned}
$$

Consequently, from (4.7) and (4.9), we have

$$
\begin{aligned}
K \widetilde{Y}_{s} & =K Y_{m} \Phi_{m, s}=X_{m+1} B_{m, m+1}^{T} \Phi_{m, s}=X_{m+1} \Psi_{m+1, s} \Sigma_{s} \\
& =X_{m+1} Q_{s+1} R_{s+1}\left[\begin{array}{c}
I_{s} \\
e_{m+1}^{T} \Psi_{m+1, s}
\end{array}\right] \Sigma_{s} \\
& =\widetilde{X}_{s+1} R_{s+1}\left[\begin{array}{c}
I_{s} \\
e_{m+1}^{T} \Psi_{m+1, s}
\end{array}\right] \Sigma_{s} .
\end{aligned}
$$

From (4.5), we know that

$$
\widetilde{Y}_{s}^{T} K M \widetilde{X}_{s+1}=\left[I_{s}, 0\right] \widetilde{B}_{s+1} \triangleq \widetilde{B}_{s, s+1},
$$

while from (4.10), we have

$$
\widetilde{X}_{s+1}^{T} K M \widetilde{Y}_{s}=R_{s+1}\left[\begin{array}{c}
I_{s} \\
e_{m+1}^{T} \Psi_{m+1, s}
\end{array}\right] \Sigma_{s} .
$$

Comparing the above two equations, it can be deduced that

$$
\widetilde{B}_{s, s+1}^{T}=R_{s+1}\left[\begin{array}{c}
I_{s} \\
e_{m+1}^{T} \Psi_{m+1, s}
\end{array}\right] \Sigma_{s}
$$

so (4.10) can be written as

$$
K \widetilde{Y}_{s}=\widetilde{X}_{s+1} \widetilde{B}_{s, s+1}^{T} .
$$

Next, let us consider the vector $K \tilde{y}_{s+1}$. Because

$$
\widetilde{X}_{s+1}^{T} M K \tilde{y}_{s+1}=\widetilde{B}_{s}^{T} \widetilde{Y}_{s+1}^{T} K \tilde{y}_{s+1}=\widetilde{B}_{s}^{T} e_{s+1}=\tilde{\alpha}_{s+1} e_{s+1},
$$

we can suppose that $K \tilde{y}_{s+1}=\tilde{\alpha}_{s+1} \tilde{x}_{s+1}+\tilde{r}_{s+1}$. Here $\tilde{r}_{s+1}$ satisfies $\tilde{X}_{s+1}^{T} M \tilde{r}_{s+1}=0$. Therefore,

$$
\begin{aligned}
K \widetilde{Y}_{s+1} & =\left[K \widetilde{Y}_{s}, K \tilde{y}_{s+1}\right]=\left[\widetilde{X}_{s+1} \widetilde{B}_{s, s+1}^{T}, \tilde{\alpha}_{s+1} \tilde{x}_{s+1}+\tilde{r}_{s+1}\right] \\
& =\widetilde{X}_{s+1}\left[\widetilde{B}_{s, s+1}^{T}, \tilde{\alpha}_{s+1} e_{s+1}\right]+\tilde{r}_{s+1} e_{s+1}^{T}=\widetilde{X}_{s+1} \widetilde{B}_{s+1}^{T}+\tilde{r}_{s+1} e_{s+1}^{T} .
\end{aligned}
$$


Let $\tilde{\beta}_{s+1}=\left\|\tilde{r}_{s+1}\right\|_{M}$ and

$$
\tilde{x}_{s+2}=\tilde{r}_{s+1} /\left\|\tilde{r}_{s+1}\right\|_{M}
$$

and set $\widetilde{X}_{s+2}=\left[\widetilde{X}_{s+1}, \tilde{x}_{s+2}\right]$ and

$$
\widetilde{B}_{s+1, s+2}=\left[\widetilde{B}_{s+1}, \tilde{\beta}_{s+1} e_{s+1}\right] .
$$

We then have

$$
K \widetilde{Y}_{s+1}=\widetilde{X}_{s+1} \widetilde{B}_{s+1}^{T}+\tilde{\beta}_{s+1} \tilde{x}_{s+2} e_{s+1}^{T}=\widetilde{X}_{s+2} \widetilde{B}_{s+1, s+2}^{T} .
$$

REMARK 4.1. $\widetilde{B}_{s+1}$ is an upper tridiagonal matrix; it is no longer an upper bidiagonal matrix like $B_{s+1}$ in WGKL and WHGKL. For simplicity, using $X_{m}, Y_{m}, B_{m}$, and $B_{m, m+1}$ to replace $\widetilde{X}_{m}, \widetilde{Y}_{m}, \widetilde{B}_{m}$, and $\widetilde{B}_{m, m+1}$, respectively, then equation (2.2) still holds for WHGKLTR.

The following is the weighted harmonic Golub-Kahan-Lanczos with thick-restarting algorithm WHGKL-TR.

ALGORITHM 5. WHGKL-TR(M,S)

1. Given an initial guess $x_{1}$ satisfying $\left\|x_{1}\right\|_{M}=1$, a tolerance tol, an integer $s$ for the number of approximate eigenvectors that we want to add to the solution subspace, an integer $m$ for the dimension of the solution subspace, as well as $\ell$ the desired number of eigenpairs. Set $\beta_{0}=1$, $y_{0}=0$;

2. Apply WGKL from the current point to generate the rest of $X_{m+1}, Y_{m}$, and $B_{m, m+1}$. If it is the first cycle, then the current point is $x_{1}$, else $x_{s+2}$;

3. Compute an $S V D$ of $B_{m, m+1}$ as in (3.6), select $\ell \leq m$ wanted singular values $\sigma_{j}$ and their associated left singular vectors $\phi_{j}$. Form $\pm \hat{\lambda}_{j}= \pm \sigma_{j}, \hat{z}_{j}^{ \pm}=\left[\begin{array}{c}\sigma_{j} X_{m} B_{m}^{-1} \phi_{j} \\ \pm Y_{m} \phi_{j}\end{array}\right]$. Use (3.8) to verify convergence; if $\left\|\mathbf{H} \hat{z}_{j}^{+}-\hat{\lambda}_{j} \hat{z}_{j}^{+}\right\|_{\mathbf{M}} \leq$ tol, then stop, else continue;

4. Generate new $\widetilde{X}_{s+2}, \widetilde{Y}_{s+1}$, and $\widetilde{B}_{s+1, s+2}$ by using the old $X_{m+1}, Y_{m}$, and $B_{m, m+1}$. Perform a QR decomposition (4.1), and use (4.2)-(4.4), (4.6), (4.11), (4.12) to generate $\widetilde{X}_{s+1}$, $\widetilde{Y}_{s}, \tilde{y}_{s+1}, \widetilde{B}_{s+1}, \tilde{x}_{s+2}$, and $\widetilde{B}_{s+1, s+2}$, respectively. Then set $X_{s+2}=\widetilde{X}_{s+2}, Y_{s+1}=\widetilde{Y}_{s+1}$, $B_{s+1, s+2}=\widetilde{B}_{s+1, s+2}$, and go to Step 2.

REMARK 4.2. In Step 3, we compute the harmonic Ritz pairs after $m$ iterations. In practice, we do the computation for each iteration $k=1, \ldots, m$. When restarting, the information chosen to add to the solution subspaces are the wanted $\ell$ singular values of $B_{m, m+1}$ with their corresponding left and right singular vectors.

5. Numerical examples. In this section, two numerical experiments are carried out by using MATLAB 8.4 (R2014b) on a laptop with an Intel Core i5-6200U CPU 2.3GHz memory 8GB under the Windows 10 operating system.

EXAMPLE 5.1. In this example, we investigate the approximate eigenvalues derived by Algorithm 2 (wGKL-1), Algorithm 3 (wHGKL), and Algorithm 4 (wGKL-2) for the LREP. The initial vector $x_{1}$ satisfied $\left\|x_{1}\right\|_{M}=1$, which is generated by using the MATLAB code randn (). There are two groups of tested matrices $K$ and $M$, all of them are symmetric positive definite. Test 1 and Test 2 are used to denote the groups, and they come from the linear response analysis for a $\mathrm{Na}_{2}$ and silane $\left(\mathrm{SiH}_{4}\right)$ compound, respectively, which are derived by the turboTDDFT code in QUANTUM ESPRESSO [6]. The order of Test 1 and Test 2 are $n=1862$ and 5660 , respectively. 

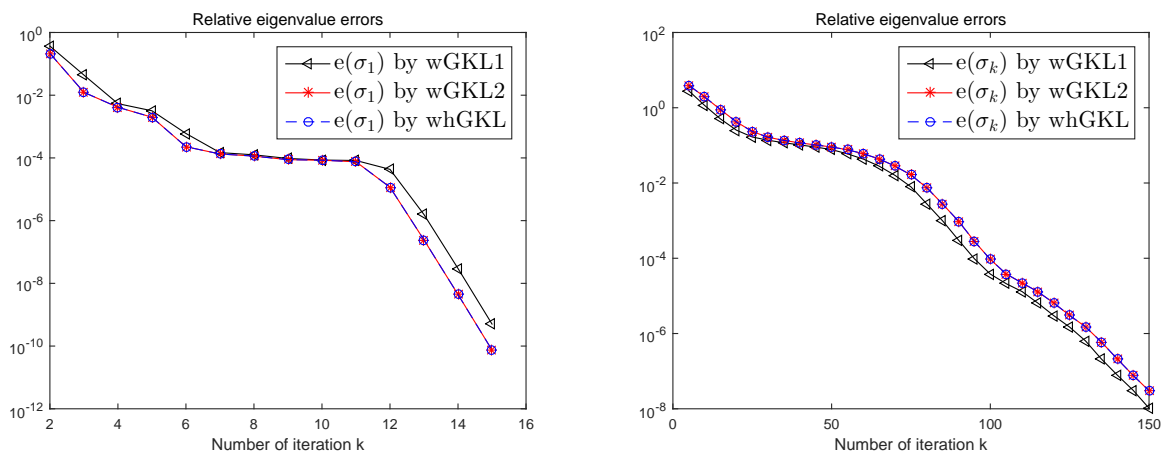

FIG. 5.1. Relative errors of the extreme positive eigenvalues for Test 1 in Example 5.1
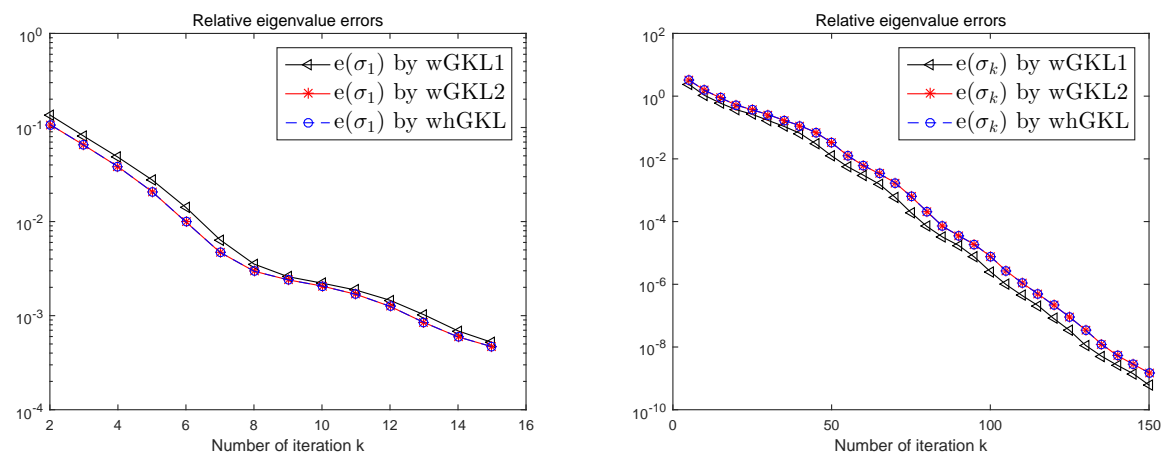

FIG. 5.2. Relative errors of the extreme positive eigenvalues for Test 2 in Example 5.1.

Suppose $\sigma_{j}$ is the $j$ th approximate eigenvalue obtained by the above three algorithms, for $j=1, \ldots, k$. We compute the relative eigenvalue errors for the largest and smallest eigenvalues of $\mathbf{H}$ :

$$
e\left(\sigma_{1}\right)=\frac{\left|\sigma_{1}-\lambda_{1}\right|}{\lambda_{1}}, \quad e\left(\sigma_{k}\right)=\frac{\left|\sigma_{k}-\lambda_{n}\right|}{\lambda_{n}}
$$

where $\lambda_{1}$ and $\lambda_{n}$ are the "exact" largest and smallest eigenvalues of $\mathbf{H}$ generated by using the MATLAB code eig ( )'.

For the largest eigenvalue, we set $k=1, \ldots, 15$, and for the smallest eigenvalue, $k=1, \ldots, 150$. In Figure 5.1 and 5.2, the numerical results are reported. From the figures, we can observe, because WGKL-2 and WHGKL both perform an SVD of the matrix $B_{k, k+1}$, that the $\sigma_{1} \mathrm{~s}$ of the two algorithms are the same in value and are closer to the largest eigenvalue of $\mathbf{H}$ than that of WGKL-1. The $\sigma_{k}$ of WGKL-1 is closest to the smallest eigenvalue of $\mathbf{H}$ among the three algorithms. Thus, the phenomena shown here are consistent with those discussed in Remark 3.9.

EXAMPLE 5.2. To test the efficiency of our methods, we compare five algorithms: Algorithm 4 (WHGKL-TR(M,S)), Algorithm 2 (WHGKL), Algorithm 1 (wGKL-1), Algorithm 3 (wGKL-2), and the first Lanczos type algorithm [21], which is labeled as ALG-TL. (Since in this paper, we only present the restarting algorithm for WHGKL, we compare with WHGKLTR and the non-restarting version of the algorithms for WGKL-1 and WGKL-2, and we 
TABLE 5.1

The matrices $K$ and $M$ in Test 3 and Test 4.

\begin{tabular}{cccc} 
Problems & $n$ & $K$ & $M$ \\
\hline Test 3 & 9604 & fv1 & finan512 \\
Test 4 & 9801 & fv2 & finan512
\end{tabular}

TABLE 5.2

Computation of the 2 largest and smallest positive eigenvalues for Test 3 and 4

\begin{tabular}{crccccccc} 
& \multicolumn{4}{c}{ Test 3} & \multicolumn{4}{c}{ Test 4} \\
\cline { 2 - 9 } Algorithms & \multicolumn{2}{c}{$\sigma_{k} \& \sigma_{k-1}$} & \multicolumn{3}{c}{$\sigma_{1} \& \sigma_{2}$} & \multicolumn{2}{c}{$\sigma_{k} \& \sigma_{k-1}$} & \multicolumn{2}{c}{$\sigma_{1} \& \sigma_{2}$} \\
& CPU & iter & CPU & iter & CPU & iter & CPU & iter \\
\hline whGKL-TR(30,5) & $\mathbf{3 . 0 2}$ & 442 & $\mathbf{0 . 5 4}$ & 82 & $\mathbf{2 . 7 9}$ & 403 & $\mathbf{0 . 4 9}$ & 75 \\
whGKL-TR(30,10) & 3.20 & 401 & 0.57 & 79 & 3.03 & 378 & 0.53 & 74 \\
whGKL-TR(30,15) & 3.60 & 390 & 0.64 & 78 & 3.43 & 371 & 0.60 & 73 \\
whGKL & 24.90 & 363 & 1.18 & 81 & 22.16 & 347 & 0.98 & 75 \\
wGKL-2 & 26.71 & 379 & 1.04 & 77 & 23.70 & 370 & 0.89 & 72 \\
wGKL-1 & 25.04 & 372 & 1.03 & 77 & 21.93 & 358 & 0.88 & 72 \\
Alg-TL & 114.37 & 372 & 1.77 & 77 & 97.54 & 358 & 1.49 & 72
\end{tabular}

expect that the three algorithms have almost the same costs in terms of time and Lanczos steps.)

We choose two pairs of symmetric positive definite matrices $K$ and $M$ for Test 3 and Test 4, and these matrices are all collected from the University of Florida sparse matrix collection [5]. Table 5.1 lists the features of the test matrices, where $n$ is the dimension of a matrix. If the dimension of the two matrices are different, we truncate the leading principal submatrix of the larger one to make $K$ or $M$ of the same size.

We aim to compute the 2 largest and smallest positive eigenvalues with their corresponding eigenvectors. If the following relative residual 1 -norm is less than $10^{-8}$, then the computed approximated eigenpair $\left(\sigma_{j}, \hat{z}_{j}\right)$ is regarded as converged,

$$
r\left(\sigma_{j}\right):=\frac{\left\|\mathbf{H} \hat{z}_{j}-\sigma_{j} \hat{z}_{j}\right\|_{1}}{\left(\|\mathbf{H}\|_{1}+\sigma_{j}\right)\left\|\hat{z}_{j}\right\|_{1}}, \quad j=1,2, k-1, k .
$$

In WHGKL-TR(M,S), we select $(m, s)$ as $(30,5),(30,10),(30,15)$, respectively, i.e., restart will occur once the dimension of the solution subspace is larger than $m$, and the information of $s$ harmonic Ritz vectors is kept. Table 5.2 reports the number of Lanczos steps labeled as "iter" and the CPU times in seconds labeled as "CPU" for the five algorithms. Bold numbers mean the least time spent.

To illustrate the accuracy of the approximations, we also compute the relative eigenvalue errors

$$
e\left(\sigma_{j}\right):= \begin{cases}\frac{\left|\lambda_{j}-\sigma_{j}\right|}{\lambda_{j}}, & j=1,2, \\ \frac{\left|\lambda_{n+j-k}-\sigma_{j}\right|}{\lambda_{n+j-k}}, & j=k-1, k,\end{cases}
$$

for Test 3, and the "exact" eigenvalues $\lambda_{j}$ are computed in the same way as in Example 5.1. The convergence curves of the relative eigenvalue error as well as the relative residual 1-norm are depicted in Figure 5.3 with $m=30, s=10$ for WHGKL-TR(M,S) and WHGKL.

Among WHGKL, WGKL-1, and WGKL-2, it is clearly demonstrated by Table 5.2 that WHGKL performs the fewest iterations for the relative residual 1-norm of the two smallest 
eigenvalues. According to the table, we also see that the number of Lanczos steps for WHGKL$\operatorname{TR}(\mathrm{M}, \mathrm{S})$ is slightly higher than for the other four non-restarted algorithms. From Figure 5.3, it can be observed that WHGKL-TR $(\mathrm{M}, \mathrm{S})$ performs a few more Lanczos steps than WHGKL to obtain the two smallest eigenvalues of Test 3 with the same accuracy. However, the computation time for WHGKL-TR $(\mathrm{M}, \mathrm{S})$ is significantly less than for the other methods. One reason is that ALG-TL must solve a $2 k \times 2 k$ eigenproblem at every iteration. Moreover, it must also sort the eigenvalues and their eigenvectors. The other algorithms only need to solve a $k \times k$ SVD problem with no sorting. In addition, reducing the $s$ orthogonalization processes in every $m$ th iterations is another reason for less time consumption. From the numerical results of WHGKL-TR $(\mathrm{M}, \mathrm{S})$ in Table 5.2, we can see that by increasing $s$, we observe a decrease in the number of iteration but the CPU time increases. This is due to the fact that the process of obtaining and adding approximate eigenvectors to the solution space takes some time. Thus, the appropriate choice for $m$ and $s$ is important for WHGKL-TR(M,S). We will investigate this in the future.
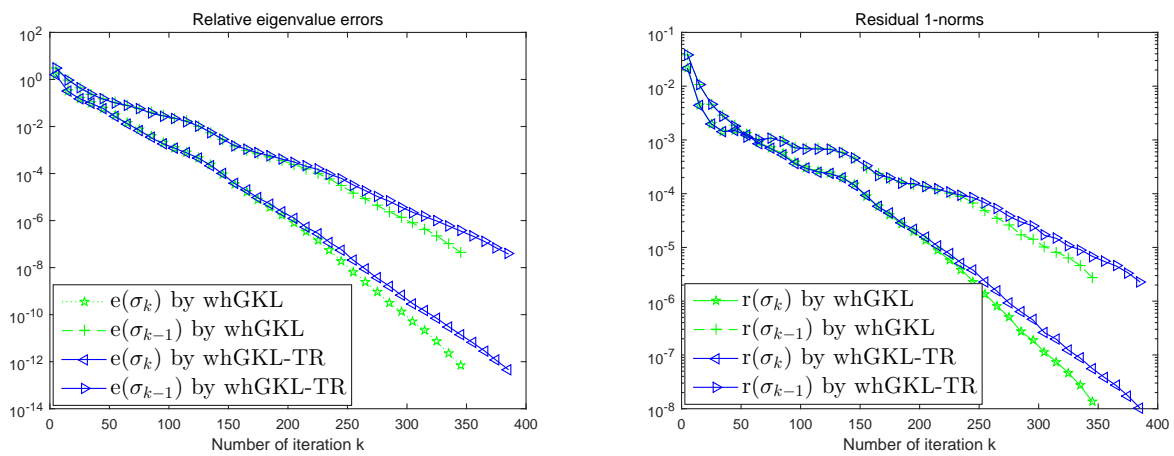

FIG. 5.3. Errors and residuals of the two smallest positive eigenvalues for Test 3 in Example 5.2

6. Conclusions. To solve the eigenvalues nearby zero for the LREP more efficiently, we have proposed a weighted harmonic Golub-Kahan-Lanczos method (WHGKL). Theoretical bounds for the harmonic Ritz pairs are established in Theorems 3.2 and 3.6. We also compare the new algorithm with the algorithms proposed in [28]. To make WHGKL more feasible, we consider to thick restart the algorithm to reduce the computational and memory costs and propose the weighted harmonic Golub-Kahan-Lanczos with thick restarting algorithm. Numerical experiments show that our new method can compute the desired eigenvalues efficiently.

Acknowledgment. The authors thank Prof. Michiel E. Hochstenbach and the anonymous referee for their useful comments and suggestions for improving the presentation of this paper. The first author was supported by the National Natural Science Foundation of China NSFC11701225, the Fundamental Research Funds for the Central Universities No. JUSRP11719, and the Natural Science Foundation of Jiangsu Province No. BK20170173. The second author was supported by the National Natural Science Foundation of China NSFC-11471122.

\section{REFERENCES}

[1] M. ARIOLI, Generalized Golub-Kahan bidiagonalization and stopping criteria, SIAM J. Matrix Anal. Appl., 34 (2013), pp. 571-592. 
[2] J. Baglama And L. ReICHEL, Augmented implicitly restarted Lanczos bidiagonalization methods, SIAM J. Sci. Comput., 27 (2005), pp. 19-42.

[3] Z. BAI AND R.-C. LI, Minimization principles for the linear response eigenvalue problem I: Theory, SIAM J. Matrix Anal. Appl., 33 (2012), pp. 1075-1100.

[4] _ Minimization principles for the linear response eigenvalue problem II: Computation, SIAM J. Matrix Anal. Appl., 34 (2013), pp. 392-416.

[5] T. A. DAVIS AND Y. Hu, The University of Florida sparse matrix collection, ACM Trans. Math. Software, 38 (2011), Art. 1, 25 pages.

[6] P. GIANNOZZI ET AL., QUANTUM ESPRESSO: A modular and open-source software project for quantum simulations of materials, J. Phys.: Condens. Matter, 21 (2009), Art. 395502, 19 pages.

[7] G. H. Golub and C. F. Van Loan, Matrix Computations, 4th ed., Johns Hopkins University Press, Baltimore, 2013.

[8] M. E. Hochstenbach, Harmonic and refined extraction methods for the singular value problem, with applications in least squares problems, BIT, 44 (2004), pp. 721-754.

[9] T.-X. LI, R.-C. LI, AND W.-W. LIN, A symmetric structure-preserving $\Gamma Q R$ algorithm for linear response eigenvalue problems, Linear Algebra Appl., 520 (2017), pp. 191-214.

[10] R. B. MoRGAN, Computing interior eigenvalues of large matrices, Linear Algebra Appl., 154/156 (1991), pp. 289-309.

[11] R. B. MorgAn AND M. ZENG, Harmonic projection methods for large non-symmetric eigenvalue problems, Numer. Linear Algebra Appl., 5 (1998), pp. 33-55.

[12] - A harmonic restarted Arnoldi algorithm for calculating eigenvalues and determining multiplicity, Linear Algebra Appl., 415 (2006), pp. 96-113.

[13] D.-T. NIU AND X.-G. YUAN, A harmonic Lanczos bidiagonalization method for computing interior singular triplets of large matrices, Appl. Math. Comput., 218 (2012), pp. 7459-7467.

[14] P. PAPAKONSTANTINOU, Reduction of the RPA eigenvalue problem and a generalized Cholesky decomposition for real-symmetric matrices, Europhys. Lett. EPL, 78 (2007), Art. 12001, 5 pages.

[15] P. Ring, Z.-Y. Ma, N. V. Giai, D. VRetenar, A. WANDELt, And L.-G. GAO, The time-dependent relativistic mean-field theory and the random phase approximation, Nuclear Phys. A, 694 (2001), pp. 249_ 268.

[16] P. Ring And P. Schuck, The Nuclear Many-Body Problem, Springer, New York, 1980.

[17] D. RoccA, Time-dependent Density Functional Perturbation Theory: New Algorithms with Applications to Molecular Spectra, PhD. Thesis, International School for Advanced Studies, Trieste, Italy, 2007.

[18] Y. SAAD, Numerical Methods for Large Eigenvalue Problems, 2nd ed., SIAM, Philadelphia, 2011.

[19] M.-Y. Shao, F. H. DA Jornada, C. YANG, J. DeslipPe, AND S. G. Louie, Structure preserving parallel algorithms for solving the Bethe-Salpeter eigenvalue problem, Linear Algebra Appl., 488 (2016), pp. 148167.

[20] G. W. STEWART, Matrix Algorithms II: Eigensystems, SIAM, Philadelphia, 2001.

[21] Z.-M. TENG AND R.-C. LI, Convergence analysis of Lanczos-type methods for the linear response eigenvalue problem, J. Comput. Appl. Math., 247 (2013), pp. 17-33.

[22] Z. TENG, Y. ZHOU, AND R.-C. LI, A block Chebyshev-Davidson method for linear response eigenvalue problems, Adv. Comput. Math., 42 (2016), pp. 1103-1128.

[23] D. J. Thouless, The Quantum Mechanics of Many-body Systems, Academic Press, New York, 1961.

[24] E. V. TsIPER, A classical mechanics technique for quantum linear response, J. Phys. B, 34 (2001), pp. L401L407.

[25] G. WU, A modified harmonic block Arnoldi algorithm with adaptive shifts for large interior eigenproblems, J. Comput. Appl. Math., 205 (2007), pp. 343-363.

[26] L.-H. ZHANG, W.-W. LIN, AND R.-C. LI, Backward perturbation analysis and residual-based error bounds for the linear response eigenvalue problem, BIT, 55 (2015), pp. 869-896.

[27] L.-H. ZHANG, J. XUE, AND R.-C. LI, Rayleigh-Ritz approximation for the linear response eigenvalue problem, SIAM J. Matrix Anal. Appl., 35 (2014), pp. 765-782.

[28] H.-X. ZHONG AND H. XU, Weighted Golub-Kahan-Lanczos bidiagonalization algorithms, Electron. Trans. Numer. Anal., 47 (2017), pp. 153-178. http://etna.ricam.oeaw.ac.at/vol.47.2017/pp153-178.dir/pp153-178.pdf

[29] H.-X. ZHONG AND G. WU, Thick restarting the weighted harmonic Arnoldi algorithm for large interior eigenproblems, Int. J. Comput. Math., 88 (2011), pp. 994-1012. 